\title{
Clinical significance of BRCA1 and BRCA2 mRNA and protein expression in patients with sporadic gastric cancer
}

\author{
HEE SUNG KIM ${ }^{1}$, IN GYU HWANG ${ }^{2}$, HYE YOUNG MIN ${ }^{3}$, YUNG-JUE BANG ${ }^{4}$ and WOO HO KIM $^{5}$ \\ Departments of ${ }^{1}$ Pathology and ${ }^{2}$ Internal Medicine, Chung-Ang University College of Medicine; \\ ${ }^{3}$ Department of Pharmacy, Chung-Ang University College of Pharmacy, Seoul 06974; Departments of \\ ${ }^{4}$ Internal Medicine and ${ }^{5}$ Pathology, Seoul National University College of Medicine, Seoul 110-799, Republic of Korea
}

Received September 27, 2018; Accepted February 8, 2019

DOI: $10.3892 / 01.2019 .10132$

\begin{abstract}
The purpose of the present study was to investigate the clinical significance of BRCA1/BRCA2 DNA repair associated (BRCA1/BRCA2) gene expression in patients with sporadic gastric cancer (GC) who had received postoperative adjuvant chemotherapy. Breast cancer type 1 and 2 susceptibility protein (BRCA1 and BRCA2) expression and BRCA1/BRCA2 mRNA expression were evaluated using immunohistochemistry (IHC) and in-situ hybridization (ISH) on tissue GC microarray tissues, in addition to reverse transcription-quantitative polymerase chain reaction (RT-qPCR). The results were analyzed for clinicopathological associations. A total of 367 cases of sporadic GC (stages II and III) were subjected to BRCA1 and BRCA2 expression analysis, and for BRCA1 and BRCA2 IHC, 360 cases were informative. A total of 61 cases (16.9\%) displayed a loss of BRCA1 and 63 (17.5\%) displayed a loss of BRCA2. BRCA1 and BRCA2 ISH results were obtained in 364 cases, of which 98 (26.9\%) presented with low expression of BRCAl mRNA and 148 (40.7\%) with low expression of BRCA2 mRNA. In 72 of the 367 cases, BRCAI and $B R C A 2$ mRNA expression levels were assessed using RT-qPCR, of which 50 (69.4\%) and 56 (77.8\%) displayed low expression of BRCA1 and BRCA2, respectively. Positive IHC expression of BRCA2 was associated with advanced tumor stage; however, BRCA1 expression was not associated with any clinicopathological parameters. Associations between the RT-qPCR and ISH methods were not significant for either $B R C A 1$ or $B R C A 2$. The results of Kaplan-Meier survival analysis with stage subgrouping revealed no significant differences with regard to survival rate. Of the multivariate analyses, neither BRCA1 nor BRCA2 IHC results were independent prognostic factors. In summary, the present study
\end{abstract}

Correspondence to: Professor Woo Ho Kim, Department of Pathology, Seoul National University College of Medicine, 28 Yeongeon-Dong, Jongno, Seoul 110-799, Republic of Korea E-mail:woohokim@snu.ac.kr

Key words: gastric cancer, breast cancer type 1 and 2 susceptibility protein, immunohistochemistry, in-situ hybridization indicated that BRCA1 and BRCA2, as assessed by IHC, may be used as clinicopathological biomarkers to evaluate the prognosis of sporadic GC.

\section{Introduction}

According to GLOBOCAN 2018 estimations, gastric cancer (GC) constitutes the sixth most common malignancy worldwide (1). Despite the decline in stomach cancer-associated mortality, its burden remains significant, being the third leading cause of cancer mortality globally. Chemotherapy serves a prominent role in the treatment of advanced- and early-stage cancer. It is therefore paramount to identify prognostic factors to assess patient responses to chemotherapy in GC.

BRCA1 DNA repair associated (BRCAl) mutations comprise risk factors for the development of breast and ovarian cancer; moreover, carriers of BRCAl germline mutations exhibit a four- to seven-fold increased risk of developing GC compared with the general population $(2,3)$. In addition, genetic alterations in the $B R C A l$ region have been identified in early-onset GC and non-specific sporadic GC (4,5); notably, BRCA1 polymorphism in sporadic GC has been reported to be associated with the response to chemotherapy (6).

The underlying mechanism of BRCA1/2 loss-of-function mutations in tumorigenesis has been linked to accelerated mutation acquisition in cells, caused by DNA double-strand breaks (DSBs) and impaired homologous recombination. Breast cancer type 1 susceptibility protein (BRCA1) and BRCA2 expression loss results in increased susceptibility to chemotherapeutic agents in patients with breast, ovarian and lung cancer (7-9). In vitro, BRCA1- and BRCA2-deficient cells display a hypersensitivity to drugs that induce DNA DSBs, including platinum-based agents $(10,11)$. The association of germline BRCA1/2 mutations with sensitivity to platinum-based agents is relatively well established in epithelial ovarian cancer (12). BRCA1/2 loss of expression may therefore be useful for predicting the efficacy of platinum-based chemotherapy in patients with GC. However, it remains controversial whether BRCA1 protein expression loss is beneficial to the survival of patients with GC (13-15). Data associated with the impact of BRCA2 expression in GC is even more limited. 
The present study aimed to investigate the role of $B R C A 1$ and $B R C A 2$ gene expression in predicting the efficacy of chemotherapy in patients with GC. BRCA1 and BRCA2 protein and mRNA expression in formalin-fixed, paraffin-embedded (FFPE) samples was evaluated using immunohistochemistry (IHC) and in-situ hybridization (ISH) on tissue microarrays (TMAs), along with reverse transcription-quantitative polymerase chain reaction (RT-qPCR) analysis of the impact of BRCA1/2 expression loss on overall survival (OS) and disease free survival (DFS).

\section{Patients and methods}

Patients. A total of 683 patients with available FFPE tumor specimens were identified, according to the confirmation of a GC diagnosis from Seoul National University College of Medicine (Seoul, South Korea) between January 2010 and December 2011. Among these patients, 365 with stage IIA-IIIC disease who underwent curative resection and available specimens for BRCA1 and BRCA2 IHC analysis were included. The inclusion criteria were as follows: i) Patients with gastric carcinoma tumor $>0.5 \mathrm{~cm}$; ii) patients who have primary gastric cancer tissue; and iii) patients with stage II and III GC. The exclusion criteria were as follows: i) Patients with gastric carcinoma tumor $<0.5 \mathrm{~cm}$; ii) patients with metastatic tumor tissue only; iii) patients with stage I or IV GC; and iv) patients who underwent palliative surgery. Adjuvant chemotherapy was administered at the discretion of the physician. Clinical data were retrieved from patient medical records, and pathological stage was reassigned according to the American Joint Committee on Cancer staging system, 7th edition (16). The median follow-up period was 71.8 months. All FFPE tissue samples were obtained from the archive of the Department of Pathology, Seoul National University Hospital (Seoul, South Korea).

The present study was approved by the Institutional Review Board (IRB)/Independent Ethics Committee of Seoul National University Hospital (H-1706-105-860). At the time of resection, consent to perform surgery was obtained from the patients, but consent for the research use of residual tissue was not obtained. The requirement for written consent was waived by the IRB due to the retrospective nature of the present study and the use of anonymous clinical data.

IHC analysis of BRCA1 and BRCA2 expression. BRCA1 and BRCA2 nuclear expression was analyzed using IHC with anti-BRCA1 (cat. no. 07-434; EMD Millipore, Billerica, MA, USA) and anti-BRCA2 (cat. no. MAB2476; R\&D Systems, Inc., Minneapolis, MN, USA) antibodies (13). For IHC, $4-\mu \mathrm{m}$ tissue sections for TMA were stained using the standard streptavidin-biotin complex method. Sectioning was performed after the specimens were de-paraffinized and rinsed in PBS. The sections were subsequently rehydrated, and antigen retrieval was performed by immersing the slides in $10 \mathrm{mM}$ citrate buffer $\mathrm{pH}$ 6.0, $0.01 \mathrm{M}$ citric acid, $0.01 \mathrm{M}$ sodium citrate) and heating for $10 \mathrm{~min}$ in the microwave. The sections were incubated for $30 \mathrm{~min}$ with non-immune serum, followed by incubation with primary mouse anti-BRCA1 (dilution, 1:150) and anti-BRCA2 (dilution, 1:500) antibodies for $2 \mathrm{~h}$. Biotinylated secondary antibody (cat. no. BP-1400; ready to use; Vector Laboratories, Inc., Burlingame, CA, USA) was added (300 $\mu \mathrm{l} / \mathrm{slide})$ for $30 \mathrm{~min}$ at room temperature, followed by streptavidin (dilution, 1:500), with diaminobenzene (DAB) as the chromogen. All sections were counterstained with hematoxylin (Sigma-Aldrich: Merck KGaA, Darmstadt, Germany) for $10 \mathrm{sec}$. Normal gastric mucosa samples were used as positive controls and samples not stained with primary antibody represented the negative controls (Fig. 1).

A modified histochemical score (H-score) was utilized to estimate BRCA1 and BRCA2 expression levels. This score included assessment of the intensity and percentage of positive cell staining. For intensity, a score index of $0,1,2$, and 3 that corresponded to negative, weak, moderate and strong staining, respectively, was utilized. The percentage of positive cells was scored as 0 to 100 . The final score was the product of intensity and positive cell percentage (range, 0-300). Positive expression of BRCA1 and BRCA2 was defined by positive staining in $\geq 10 \%$ of the cancer cells. Positive expression of BRCA1 and BRCA2 was further classified as low and high expression according to the median $\mathrm{H}$-score cut-off of expression. Median $\mathrm{H}$-score was 60 for BRCA1 and 70 for BRCA2.

RNA ISH. In situ detection of BRCAI and BRCA2 mRNA transcription using the RNAscope kit (Advanced Cell Diagnostics, Hayward, CA, USA) was performed according to the manufacturer's protocol. Housekeeping gene Peptidylpropyl isomerase B (PPIB) was used as an internal-control mRNA; if the PPIB score was 0 , the sample was regarded as not available for gene expression. A horseradish peroxidase-based signal amplification system was used for hybridization to the target probes, followed by color development with 3,3'-diaminobenzidine. The slides were counter-stained with hematoxylin for $10 \mathrm{sec}$ at room temperature. Positive staining was determined by brown punctate dots in the nucleus and/or cytoplasm. BRCA1 and BRCA2 expression was scored using the instructions in the RNAscope FFPE assay kit: Score 0, no staining; score 1, staining that was difficult to identify under a 40X objective lens in $>10 \%$ of tumor cells; score 2, staining that was difficult to identify under a 20X objective lens, but easy under a 40X objective lens in $>10 \%$ of tumor cells; score 3 , staining that was difficult to identify under a $10 \mathrm{X}$ objective lens, but easy under a $20 \mathrm{X}$ objective lens in $>10 \%$ of tumor cells; and score 4 , easy to identify under a 10X objective lens in $>10 \%$ of tumor cells.

$R T-q P C R$. A total of $110 \mathrm{GC}$ specimens were obtained for mRNA extraction. Of these specimens, RNA from 72 was successfully amplified. Tumor tissue from a representative paraffin block was cut into $10-\mu \mathrm{m}$ sections, and micro-dissection was utilized to minimize the influence on the surrounding normal tissues. The proportion of tumor cells was $\sim 80 \%$. Total RNA was extracted from the FFPE tumor tissue using the RNeasy FFPE kit (Qiagen GmbH, Hilden, Germany) according to the manufacturer's protocol. Total RNA (1 mg) was reverse transcribed using the GoScript reverse transcription system (Promega Corporation, Madison, WI, USA) according to the manufacturer's protocol.

Template cDNA was amplified using the TaqMan ${ }^{\circledR}$ gene expression assay (Applied Biosystems, Foster City, CA, USA) and the 7500 PCR system (Applied Biosystems; Thermo 
Fisher Scientific, Inc.). The 20- $\mu$ l PCR mixture contained 2X Premix ExTaq (Probe qPCR; Takara Bio, Inc., Otsu, Japan), $1 \mu \mathrm{l}$ cDNA and $1 \mu \mathrm{l}$ each of the primers and probes [BRCA1 (Hs01556193_m1), BRCA2 (Hs00609073_m1), and ACTB (Hs99999903_m1) (cat. no. 4331182)]. Amplification was performed in a 96 -well optical plate at $95^{\circ} \mathrm{C}$ for $30 \mathrm{sec}$, followed by 45 cycles at $95^{\circ} \mathrm{C}$ for $5 \mathrm{sec}$ and $60^{\circ} \mathrm{C}$ for $34 \mathrm{sec}$. The experiment was performed in triplicate, and relative gene expression was calculated according to the comparative $\mathrm{Cq}$ method (17). The final values were determined using the following formula: mRNA expression level $=2^{-\Delta \Delta \mathrm{Cq}}$.

Statistical analysis. All statistical analyses were performed using SPSS statistics 23 software (IBM Corp., Armonk, NY, USA). Differences in clinicopathological parameters according to nuclear BRCA1 and BRCA2 expression were evaluated using the $\chi^{2}$ test and linear-by-linear association. The minimum, maximum and mean values of relative BRCA1 and BRCA2 mRNA expression were calculated as $2^{-\Delta \Delta C q}$ of each gene and plotted in Fig. 2. OS was defined from initial cancer diagnosis to the time of death (from any cause). Patient survival was analyzed using a Kaplan-Meier plot and the log-rank test. The period of disease-free survival (DFS) was defined as the duration between surgery and disease recurrence, any cause of death prior to disease recurrence, or the most recent follow-up. Comparison of the methods used to measure BRCA1 and BRCA2 expression levels was performed using the $\chi^{2}$ test. $\mathrm{P}<0.05$ was considered to indicate a statistically significant difference.

\section{Results}

Patient characteristics and clinical outcomes. In the present study, a total of 367 patient samples were analyzed and baseline characteristics are summarized in Table I. The median age was $62 \pm 12.9$ years. A total of 253 patients $(68.9 \%)$ underwent subtotal gastrectomy and 114 patients $(31.1 \%)$ underwent total gastrectomy. Neoadjuvant chemotherapy was administered to 9 patients. Of the 273 informative cases, 180 (49.0\%) received postoperative chemotherapy. The chemotherapy regimen were as follows: Etoposide and platinum agent $(n=3)$; fludarabine and mitoxantrone $(\mathrm{n}=1)$; 5-fluorouracil (5-FU), leucovorin (folinic acid) and oxaliplatin $(n=1) ; 5-F U$ and cisplatin $(n=67) ; F U$ $(n=12)$; tegafur, gimeracil, and oteracil potassium (S-1) $(n=62)$; S-1 with cisplatin $(n=6)$; capecitabine and oxaliplatin $(n=17)$; and capecitabine and cisplatin $(n=28)$. Of the 192 informative cases, a platinum regimen was used in 135 patients.

Adjuvant chemotherapy was used by $43.5 \%$ of patients $(n=77)$ with stage II disease and in $54.2 \%(n=103)$ with stage III disease. In $22 \%$ of patients $(n=39)$ with stage II disease and $50 \%$ of patients $(n=95)$ with stage III disease, a platinum-based treatment was included in the adjuvant chemotherapy regimen. Histological grades according to WHO classification of well differentiated, moderately differentiated and poorly differentiated were represented by $6.0,30.0$ and $47.1 \%$ of patients, respectively. Intestinal type (according to Lauren classification) was observed in $40.3 \%$ of patients. Lymphatic, venous and perineural invasion were identified in $67.0,22.9$ and $53.1 \%$ of patients, respectively. Relapse occurred in 76 patients $(20.7 \%)$ and mortality in
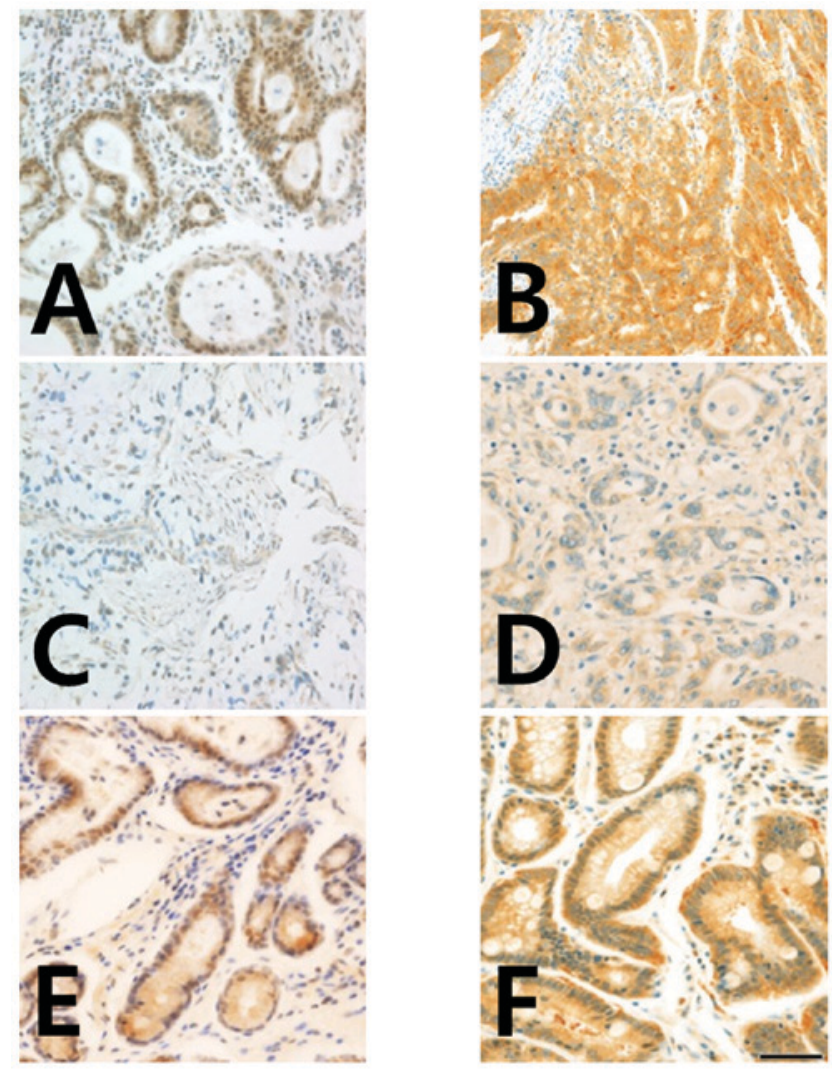

Figure 1. Immunohistochemistry of BRCA1 and BRCA2 protein expression in gastric cancer tissues. (A) Strong positive BRCA1 staining in the cytoplasm and nuclei, and (B) positive BRCA2 staining in the cytoplasm of gastric adenocarcinoma cells. (C) Negative BRCA1 staining in the cytoplasm and nuclei, and (D) negative BRCA2 staining in the nucleus and cytoplasm of gastric adenocarcinoma cells. (E) Positive BRCA1 staining in the cytoplasm, and $(\mathrm{F})$ positive BRCA2 staining in the cytoplasm of chronic gastritis cells. Magnification, $\mathrm{x} 400$ (scale bar, $50 \mu \mathrm{m}$ ). BRCA1/2, breast cancer type 1 and 2 susceptibility protein.

102 patients $(29.2 \%)$ during the median follow-up period of 44.2 months. The 5-year DFS rate was $70.0 \%$ and the 5-year OS rate was $66.0 \%$.

A total of 360 of the $367 \mathrm{GC}$ cases were informative for BRCA1 and BRCA2 IHC. A lack of BRCA1 expression was observed in 61 patients (16.9\%) and BRCA1-positive expression was observed in 299 patients $(83.1 \%)$. No clinicopathological parameters were significantly associated with BRCA1 expression status (Table II). A lack of BRCA2 expression was observed in 63 patients (17.5\%) and BRCA2-positive expression was apparent in 296 patients (82.4\%). A lack of BRCA2 expression was associated with patient age $<45$ years $(\mathrm{P}=0.01)$, a histological type of signet ring cell carcinoma $(\mathrm{P}<0.001)$ and a Lauren classification of diffuse type $(\mathrm{P}<0.001)$.

Prognosis according to BRCA1 and BRCA2 expression. Following Kaplan-Meier analysis, neither BRCA1 nor BRCA2 (as evaluated by IHC) had any significant impact on the OS or DFS of patients with stage II/III GC. Total patient and subgroup analyses of DFS and OS did not highlight any significant differences between patients who received adjuvant chemotherapy and those who did not (Fig. 2). The benefits of adjuvant chemotherapy and platinum regimens were not statistically significant, although platinum-treated 
Table I. Patient characteristics.

\begin{tabular}{|c|c|c|}
\hline Variable & $\mathrm{n}$ & $\%$ \\
\hline \multicolumn{3}{|l|}{ Sex } \\
\hline Male & 239 & 65.1 \\
\hline Female & 128 & 34.9 \\
\hline \multicolumn{3}{|l|}{ Age, years } \\
\hline$\leq 45$ & 45 & 12.3 \\
\hline $45<x \leq 60$ & 130 & 35.4 \\
\hline$>60$ & 192 & 52.3 \\
\hline \multicolumn{3}{|l|}{ Adjuvant chemotherapy } \\
\hline No & 93 & 25.3 \\
\hline Yes & 180 & 49.0 \\
\hline Not available & 94 & 25.6 \\
\hline \multicolumn{3}{|l|}{ Histological grade and type } \\
\hline Well differentiated & 22 & 6.0 \\
\hline Moderately differentiated & 110 & 30.0 \\
\hline Poorly differentiated & 173 & 47.1 \\
\hline Signet ring cell carcinoma & 48 & 13.1 \\
\hline Mucinous adenocarcinoma & 13 & 3.5 \\
\hline Undifferentiated carcinoma & 1 & 0.3 \\
\hline \multicolumn{3}{|l|}{ T-stage ${ }^{a}$} \\
\hline $\mathrm{T} 1$ & 19 & 5.2 \\
\hline $\mathrm{T} 2$ & 49 & 13.4 \\
\hline $\mathrm{T} 3$ & 183 & 49.9 \\
\hline $\mathrm{T} 4$ & 116 & 31.6 \\
\hline \multicolumn{3}{|l|}{$\mathrm{N}$-stage ${ }^{\mathrm{a}}$} \\
\hline N0 & 78 & 21.3 \\
\hline N1 & 84 & 22.9 \\
\hline $\mathrm{N} 2$ & 100 & 27.2 \\
\hline N3 & 105 & 28.6 \\
\hline \multicolumn{3}{|l|}{ TNM stage ${ }^{a}$} \\
\hline II & 177 & 48.2 \\
\hline III & 190 & 51.8 \\
\hline \multicolumn{3}{|l|}{ Lymphatic invasion } \\
\hline Absent & 121 & 33 \\
\hline Present & 246 & 67 \\
\hline \multicolumn{3}{|l|}{ Venous invasion } \\
\hline Absent & 283 & 77.1 \\
\hline Present & 84 & 22.9 \\
\hline \multicolumn{3}{|l|}{ Perineural invasion } \\
\hline Absent & 172 & 46.9 \\
\hline Present & 195 & 53.1 \\
\hline
\end{tabular}

${ }^{\mathrm{a} A s}$ defined by the American Joint Committee on Cancer 7th edition; TNM, Tumor-Node-Metastasis.

BRCA1-positive patients with stage II disease exhibited a longer DFS time ( $\mathrm{P}=0.016$; Table III). The prognostic value of BRCA1 and BRCA2 expression did not remain significant. Vascular invasion $(\mathrm{P}=0.028)$, perineural invasion $(\mathrm{P}=0.001)$ and tumor stage $(\mathrm{P}<0.001)$ were significantly associated with DFS. Perineural invasion $(\mathrm{P}=0.004)$, adjuvant chemotherapy
$(\mathrm{P}=0.001)$ and tumor stage $(\mathrm{P}<0.001)$ were significantly associated with OS (Table IV).

BRCA1 and BRCA2 $m R N A$ and protein expression. Positive BRCA1 IHC staining was observed in the cytoplasm and nuclei of tumor cells, whereas BRCA2 was only detected in the cytoplasm of GC cells (Fig. 1). A lack of BRCA2 expression has been associated with advanced Tumor-Node-Metastasis (TNM) stage. BRCA1 and BRCA2 mRNA from 72 of the 110 cases were successfully amplified using RT-qPCR (Fig. 3). Comparisons between the IHC, ISH and RT-qPCR results were made by pairwise tabulation of BRCA 1 and BRAC2 ISH scores in columns, and BRCA1 and BRAC2 IHC, and BRCA1 and BRAC2 RT-qPCR in rows (Table V). The results demonstrated a significant association between BRCA1 mRNA expressions measured by RT-qPCR and ISH score $(\mathrm{P}=0.009)$ and between BRCA2 mRNA expressions measured by RT-qPCR and ISH score $(\mathrm{P}=0.051)$.

\section{Discussion}

BRCA1, an essential component of numerous DNA damage repair pathways, and pathways involved in cellular responses to microtubule damage, is considered to be a differential modulator of cancer survival following treatment with cisplatin and taxanes. In particular, preclinical and clinical studies have reported that BRCA1 expression level is associated with cisplatin and taxane chemosensitivity (13). Moreover, germline mutations in BRCAl and $B R C A 2$ are detected in $14.5 \%$ of serous ovarian carcinomas. However, in epithelial ovarian cancer, a more favorable prognosis has been demonstrated for those with BRCA1 and $B R C A 2$ mutations (4). Although the mechanism driving the association between $B R C A 1 / 2$ mutations and survival is not known, it has been suggested that the survival advantage of mutation may be mediated by an improved response to platinum-based agents (5).

Improved practical methods to determine BRCA1 and BRCA 2 mRNA and protein expression are currently required. Therefore, the present study utilized IHC, ISH and RT-qPCR to detect $B R C A$ gene expression in FFPE GC tissues, and evaluated the clinical relevance of these genes in sporadic GC. In breast and ovarian cancer, it has been illustrated that mRNA expression of BRCA1 and BRCA2 has prognostic value and clinical impact in terms of chemotherapeutic response (18). From the identification of a reliable antibody for BRCA1 protein IHC through the evaluation of the specificity and sensitivity of available anti-BRCA1 antibodies (19), BRCA1 expression was revealed to be associated with certain molecular events in ovarian high-grade serous carcinomas, with no cases of $B R C A 1$ germline mutation displaying intact immunostaining (negative predictive value, 100\%) (20). Moreover, IHC analysis is considered the most effective approach to stratify patients with high-grade serous ovarian carcinoma for germline genetic testing (20). By contrast, the frequency of $B R C A 1$ mutations among patients with breast cancer is $<5 \%$ (21), whereas the loss of BRCA1 protein expression is $\sim 20 \%$ (22). From a clinical perspective, IHC is also the most accurate method for identifying the loss of gene expression. In the present study, although no significant 
Table II. Association between BRCA immunohistochemistry status and clinicopathological features in 360 informative gastric cancer cases.

\begin{tabular}{|c|c|c|c|c|c|c|c|c|c|c|c|c|}
\hline \multirow[b]{3}{*}{ Variable } & \multicolumn{4}{|c|}{ BRCA1 IHC } & \multirow[b]{3}{*}{ P-value } & \multicolumn{4}{|c|}{ BRCA2 IHC } & \multirow[b]{3}{*}{ P-value } & \multirow{2}{*}{\multicolumn{2}{|c|}{ Total }} \\
\hline & \multicolumn{2}{|c|}{ Negative } & \multicolumn{2}{|c|}{ Positive } & & \multicolumn{2}{|c|}{ Negative } & \multicolumn{2}{|c|}{ Positive } & & & \\
\hline & $\mathrm{n}$ & $\%$ & $\mathrm{n}$ & $\%$ & & $\mathrm{n}$ & $\%$ & $\mathrm{n}$ & $\%$ & & $\mathrm{n}$ & $\%$ \\
\hline Total patients & 61 & 16.9 & 299 & 83.1 & & 63 & 17.5 & 297 & 82.5 & & & \\
\hline Sex & & & & & 0.459 & & & & & 0.045 & & \\
\hline Male & 42 & 18.0 & 191 & 82.0 & & 34 & 14.6 & 199 & 85.4 & & 233 & 64.7 \\
\hline Female & 19 & 15.0 & 108 & 85.0 & & 29 & 23.0 & 97 & 77.0 & & 127 & 35.3 \\
\hline Age, years & & & & & 0.018 & & & & & 0.220 & & \\
\hline$<45$ & 4 & 9.3 & 39 & 90.7 & & 11 & 25.6 & 32 & 74.4 & & 43 & 11.9 \\
\hline $45-60$ & 15 & 11.7 & 113 & 88.3 & & 24 & 18.8 & 104 & 81.3 & & 128 & 35.6 \\
\hline$>60$ & 42 & 22.2 & 147 & 77.8 & & 28 & 14.8 & 161 & 85.2 & & 189 & 52.5 \\
\hline Histological grade and type & & & & & 0.174 & & & & & $<0.001$ & & \\
\hline Well differentiated & 3 & 13.6 & 19 & 86.4 & & 2 & 9.1 & 20 & 90.9 & & 22 & 6.1 \\
\hline Moderately differentiated & 25 & 23.4 & 82 & 76.6 & & 9 & 8.4 & 98 & 91.6 & & 107 & 29.7 \\
\hline Poorly differentiated & 29 & 16.9 & 143 & 83.1 & & 27 & 15.7 & 145 & 84.3 & & 172 & 47.8 \\
\hline Signet ring cell carcinoma & 3 & 6.5 & 43 & 93.5 & & 21 & 46.7 & 24 & 53.3 & & 46 & 12.8 \\
\hline Mucinous adenocarcinoma & 1 & 8.3 & 11 & 91.7 & & 4 & 33.3 & 8 & 66.7 & & 12 & 3.3 \\
\hline Undifferentiated carcinoma & 0 & 0.0 & 1 & 100.0 & & 0 & 0.0 & 1 & 100.0 & & 1 & 0.3 \\
\hline Lauren classification & & & & & 0.065 & & & & & $<0.001$ & & \\
\hline Intestinal & 31 & 21.4 & 114 & 78.6 & & 13 & 9.0 & 132 & 91.0 & & 145 & 40.3 \\
\hline Diffuse & 30 & 14.0 & 185 & 86.0 & & 50 & 23.4 & 164 & 76.6 & & 215 & 59.7 \\
\hline Location & & & & & 0.056 & & & & & 0.072 & & \\
\hline Upper third & 13 & 19.1 & 55 & 80.9 & & 9 & 13.2 & 59 & 86.8 & & 68 & 18.9 \\
\hline Middle third & 8 & 8.8 & 83 & 91.2 & & 23 & 25.3 & 68 & 74.7 & & 91 & 25.3 \\
\hline Lower third & 40 & 19.9 & 161 & 80.1 & & 31 & 15.4 & 170 & 84.6 & & 201 & 55.8 \\
\hline T stage & & & & & 0.739 & & & & & 0.072 & & \\
\hline $\mathrm{T} 1$ & 4 & 22.2 & 14 & 77.8 & & 6 & 33.3 & 12 & 66.7 & & 18 & 5.0 \\
\hline $\mathrm{T} 2$ & 10 & 20.8 & 38 & 79.2 & & 4 & 8.3 & 44 & 91.7 & & 48 & 13.3 \\
\hline $\mathrm{T} 3$ & 30 & 16.8 & 149 & 83.2 & & 29 & 16.3 & 149 & 83.7 & & 179 & 49.7 \\
\hline $\mathrm{T} 4$ & 17 & 14.8 & 98 & 85.2 & & 24 & 20.9 & 91 & 79.1 & & 115 & 31.9 \\
\hline $\mathrm{N}$ stage & & & & & 0.582 & & & & & 0.796 & & \\
\hline No & 9 & 11.8 & 67 & 88.2 & & 14 & 18.7 & 61 & 81.3 & & 76 & 21.1 \\
\hline N1 & 14 & 17.1 & 68 & 82.9 & & 15 & 18.3 & 67 & 81.7 & & 82 & 22.8 \\
\hline $\mathrm{N} 2$ & 19 & 19.4 & 79 & 80.6 & & 14 & 14.3 & 84 & 85.7 & & 98 & 27.2 \\
\hline N3 & 19 & 18.3 & 85 & 81.7 & & 20 & 19.2 & 84 & 80.8 & & 104 & 28.9 \\
\hline TNM stage & & & & & 0.810 & & & & & 0.580 & & \\
\hline II & 30 & 17.4 & 142 & 82.6 & & 32 & 18.7 & 139 & 81.3 & & 172 & 47.8 \\
\hline III & 31 & 16.5 & 157 & 83.5 & & 31 & 16.5 & 157 & 83.5 & & 188 & 52.2 \\
\hline Lymphatic invasion & & & & & 0.725 & & & & & 0.280 & & \\
\hline Absent & 21 & 17.9 & 96 & 82.1 & & 24 & 20.7 & 92 & 79.3 & & 117 & 32.5 \\
\hline Present & 40 & 16.5 & 203 & 83.5 & & 39 & 16.0 & 204 & 84.0 & & 243 & 67.5 \\
\hline Vascular invasion & & & & & 0.358 & & & & & 0.120 & & \\
\hline Absent & 44 & 15.9 & 232 & 84.1 & & 53 & 19.3 & 222 & 80.7 & & 276 & 76.7 \\
\hline Present & 17 & 20.2 & 67 & 79.8 & & 10 & 11.9 & 74 & 88.1 & & 84 & 23.3 \\
\hline Perineural invasion & & & & & 0.066 & & & & & 0.673 & & \\
\hline Absent & 35 & 20.8 & 133 & 79.2 & & 31 & 18.5 & 137 & 81.5 & & 168 & 46.7 \\
\hline Present & 26 & 13.5 & 166 & 86.5 & & 32 & 16.8 & 159 & 83.2 & & 192 & 53.3 \\
\hline
\end{tabular}

BRCA1/2, Breast cancer type 1 and 2 susceptibility protein; TNM, Tumor-Node-Metastasis; IHC, immunohistochemistry. 

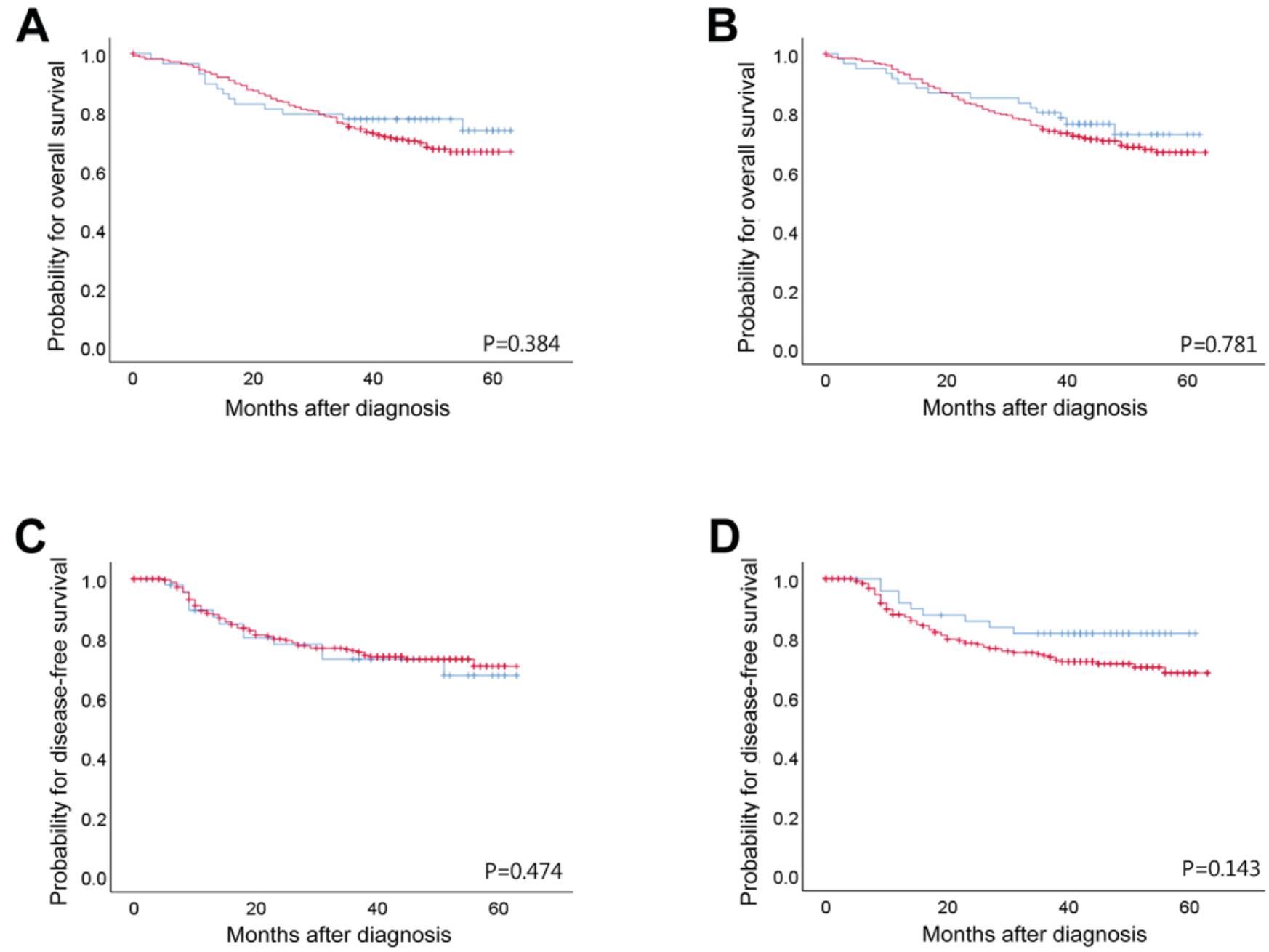

Figure 2. Kaplan-Meier survival analysis of patients with gastric cancer. Overall survival according to (A) BRCA1 IHC and (B) BRCA2 IHC. Disease free survival according to (C) BRCA1 IHC and, (D) BRCA2 IHC. Blue line; negative expression of BRCA1/BRCA2 protein by IHC, red line; positive expression of BRCA1/BRCA2 protein by IHC. BRCA1/2, breast cancer type 1 and 2 susceptibility protein; IHC, immunohistochemistry.

association was identified between methods, the findings verified that IHC determination of BRCA protein expression represents a suitable method of detection in GC specimens. Moreover, IHC evaluation of BRCA2 expression levels was more clinically significant when compared with BRCA1. By contrast, other studies have illustrated that BRCA1 expression (evaluated by IHC) is more closely associated with clinical outcome $(2,13,23)$.

Previously, Wang et al (23) studied a large cohort of patients with cancer of the digestive system, including 627 stomach, 442 intestinal, 255 liver and 222 pancreatic cancer cases. BRCA1 and BRCA2 expression was determined using IHC, and associations between clinicopathological parameters and patient survival were determined. The study revealed downregulation of cytoplasmic BRCA1 and BRCA2, and upregulation of nuclear BRCA1 in digestive tumors. Low expression levels of cytoplasmic BRCA1 and BRCA2, and high expression levels of nuclear BRCA1 were commonly associated with advanced TNM stage. Cytoplasmic BRCA1 and BRCA2 were independent favorable prognostic factors in gastric, colorectal and liver cancer, and nuclear BRCA1 expression was an independent prognostic factor predicting poor outcome in all of the cancer types investigated.
By contrast, the present study involved 367 cases of gastric cancer, and the IHC data were limited to revealing a relatively close association between a lack of BRCA2 expression and higher T stage. One of the aims of the study was to evaluate the efficiency of the ISH method in determining BRCA1 and BRCA2 mRNA expression levels in GC tissues, when compared with RT-qPCR. Although not statistically significant, a link between BRCA1 expression levels determined by ISH and RT-qPCR was demonstrated.

A number of studies used fresh tissues to quantify BRCA1 mRNA expression levels with RT-qPCR $(14,18)$, although there is no evidence of previous studies performing a paired evaluation of BRCA1 mRNA using ISH and RT-qPCR (24). Due to the effects of FFPE processing, the present study was limited in terms of mRNA quality; BRCA1 and BRCA2 mRNA were successfully amplified in only 72 of the 110 submitted cases, which was considered to have influenced the lack of statistical significance between the results generated using ISH and RT-qPCR.

BRCA1 protein is known to serve as a therapeutic target for DNA-damaging chemotherapy in breast, ovarian and lung cancer treatment $(25,26)$. The loss or reduction of BRCA1 mRNA and protein expression has been demonstrated 
Table III. Comparison of probability values of Kaplan-Meier survival analyses for stage II and III ( $\mathrm{n}=343)$, stage II ( $\mathrm{n}=160)$ and stage III $(n=183)$ patients.

\begin{tabular}{|c|c|c|c|c|c|c|c|c|c|c|c|c|}
\hline \multirow[b]{3}{*}{ Treatment } & \multicolumn{6}{|c|}{ P-value for BRCA1 IHC } & \multicolumn{6}{|c|}{ P-value for BRCA2 IHC } \\
\hline & \multicolumn{3}{|c|}{ OS } & \multicolumn{3}{|c|}{ DFS } & \multicolumn{3}{|c|}{ OS } & \multicolumn{3}{|c|}{ DFS } \\
\hline & II+III & II & III & II+III & II & III & $\mathrm{II}+\mathrm{III}$ & II & III & II+III & II & III \\
\hline All patients & 0.384 & 0.752 & 0.513 & 0.781 & 0.355 & 0.605 & 0.474 & 0.386 & 0.738 & 0.143 & 0.262 & 0.398 \\
\hline Adjuvant chemotherapy & 0.146 & 0.475 & 0.318 & 0.549 & 0.144 & 0.75 & 0.205 & 0.25 & 0.394 & 0.146 & 0.336 & 0.318 \\
\hline No adjuvant chemotherapy & 0.597 & 0.336 & 0.633 & 0.503 & 0.822 & 0.392 & 0.539 & 0.952 & $0.007^{\mathrm{a}}$ & 0.597 & 0.475 & 0.633 \\
\hline Platinum regimen & 0.374 & $0.05^{\mathrm{b}}$ & 0.253 & 0.329 & 0.016 & 0.518 & 0.137 & 0.502 & 0.228 & 0.11 & 0.977 & 0.141 \\
\hline Non-platinum regimen & 0.242 & 0.586 & 0.349 & 0.148 & 0.378 & 0.281 & 0.528 & 0.488 & 0.984 & 0.796 & 0.272 & 0.175 \\
\hline
\end{tabular}

Table IV. Multivariate analysis of factors associated with DFS and OS.

\begin{tabular}{|c|c|c|c|c|c|c|}
\hline \multirow[b]{2}{*}{ Variable } & \multicolumn{3}{|c|}{ DFS } & \multicolumn{3}{|c|}{ OS } \\
\hline & Hazard ratio & $95 \% \mathrm{CI}$ & P-value & Hazard ratio & $95 \% \mathrm{CI}$ & P-value \\
\hline BRCA1 IHC-negative & 1.625 & $0.854-3.093$ & 0.139 & 0.823 & $0.427-1.588$ & 0.562 \\
\hline BRCA2 IHC-negative & 0.668 & $0.316-1.413$ & 0.292 & 0.971 & $0.473-1.996$ & 0.937 \\
\hline Age $<60$ years & 0.503 & $0.464-1.458$ & 0.503 & 0.769 & $0.426-1.386$ & 0.382 \\
\hline Lymphatic invasion & 1.205 & $0.633-2.293$ & 0.57 & 1.257 & $0.662-2.386$ & 0.485 \\
\hline Vascular invasion & 1.826 & $1.068-3.121$ & 0.028 & 1.28 & $0.754-2.174$ & 0.361 \\
\hline Perineural invasion & 2.704 & $1.477-4.95$ & 0.001 & 2.234 & $1.294-3.856$ & 0.004 \\
\hline Adjuvant chemotherapy & 0.834 & $0.422-1.652$ & 0.603 & 0.349 & $0.193-0.633$ & 0.001 \\
\hline Stage III vs. stage II & 3.564 & $1.828-6.946$ & $<0.001$ & 4.689 & $2.388-9.211$ & $<0.001$ \\
\hline
\end{tabular}

BRCA1/2, breast cancer type 1 and 2 susceptibility protein; IHC, immunohistochemistry; CI, confidence interval; DFS, disease-free survival; OS, overall survival.

in sporadic breast cancer, and its prognostic significance has been reported $(24,27)$. Other DNA repair-associated biomarkers include ataxia telangiectasia mutated (ATM), ATM-Rad3-related gene, mediator of DNA damage checkpoint protein 1 and meiotic recombination 11 (12) along with DNA repair-associated biomarkers induced by alcohol, including transcription factor IIB-related factor 1 and myeloperoxidase (28). In non-small cell lung cancer and GC patients with malignant effusions, excision repair cross-complementing group 1 and BRCAl mRNA expression levels correlated with in vitro chemosensitivity to cisplatin and/or docetaxel (4).

BRCA1 and BRCA2 serve multi-aspect roles in a number of DNA repair pathways, including those involved in homologous recombination, non-homologous end joining and single-strand annealing. During homologous recombination, BRCA1 acts as a linker between the detection of DNA damage and the effectors that execute DSB repair (29), whereas BRCA2 primarily triggers DSB repair through its interaction with DNA repair protein RAD51 homolog 2 (30). When the homologous recombination pathway is impaired, DSBs can result in genomic instability, which further predisposes individuals to cancer.

In the present study, the loss of BRCA1 and BRCA2 expression correlated with certain clinicopathological parameters in a cohort of Korean patients. The effects of platinum-based agents in postoperative adjuvant chemotherapy was also evaluated. During conventional adjuvant chemotherapy, platinum-based agents are administered to patients at higher clinical stages who are at a higher risk. However, no statistical significance was demonstrated between the expression of BRCA1 or BRCA2 and survival outcome. The retrospective nature of the study was also somewhat limiting, and a randomized prospective study is required to remove confounding factors and subsequent bias.

In conclusion, the present study revealed that BRCA1 and BRCA2, as evaluated by IHC, constitute clinically useful biomarkers for the detection of sporadic GC. A randomized prospective clinical trial is necessary to evaluate the effects of 


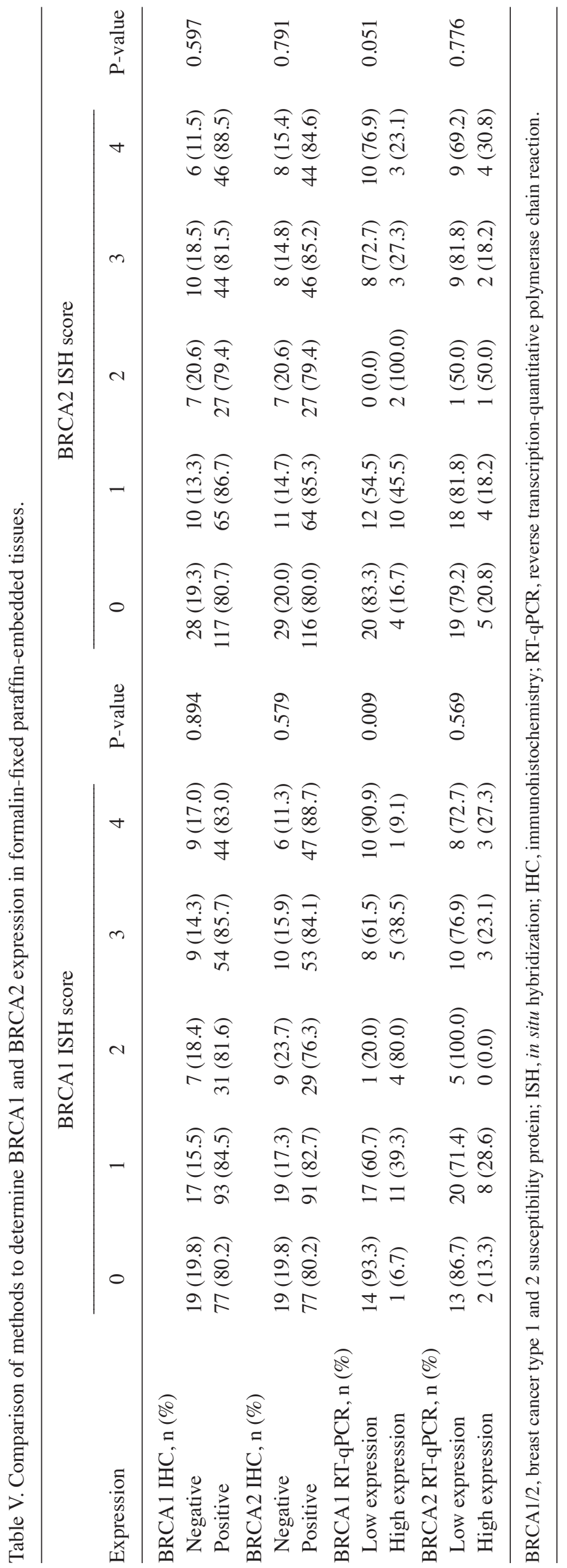




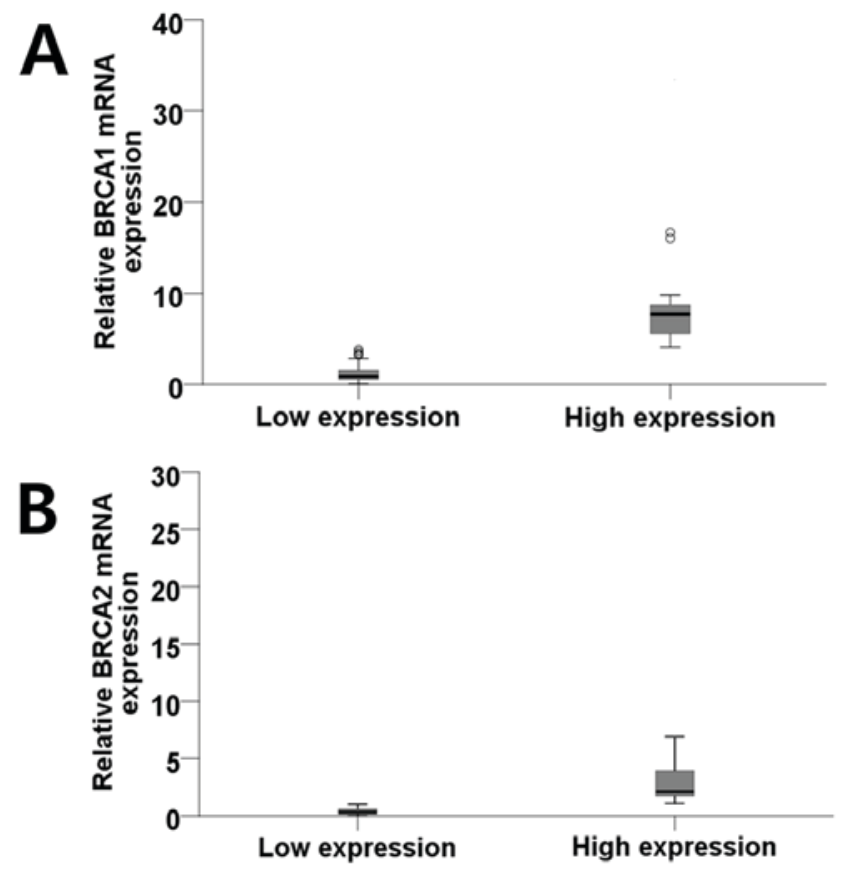

Figure 3. (A) Diagram illustrating the distribution of patients according to the mean value (3.90) of the BRCA1 mRNA expression level into low (0.06-3.84; mean, 1.27) and high (4.10-33.26; mean, 9.53) groups (outliners: Open circle, $>1.5 x$ interquartile range; asterisk, $>3 x$ interquartile range). (B) Diagram illustrating the distribution of patients according to the mean value (1.00) of the BRCA1 mRNA expression level into low (0.04-1.00; mean, 0.38) and high (1.10-25.42; mean, 4.19) groups (outliners: Open circle, $>1.5 \mathrm{X}$ interquartile range). BRCA1/2, BRCA1/2 DNA repair associated.

BRCA1 and BRCA2 expression on therapeutic sensitivity, and the benefits of adjuvant chemotherapy or platinum regimens in patients with GC.

\section{Acknowledgements}

The authors would like to thank Ms Hye Jung Lee and Ms Seung Hee Jung in Seoul National University College of Medicine, Seoul Korea for construction of the tissue array, and for assistance in performing the IHC staining and ISH.

\section{Funding}

The present study was supported by a National Research Foundation of Korea grant funded by the Korean government (grant no. 2014R1A1A2057925).

\section{Availability of data and materials}

The datasets used and/or analyzed during the current study are available from the corresponding author on reasonable request.

\section{Authors' contributions}

HSK evaluated the IHC slides and ISH, performed RT-qPCR and wrote the manuscript. IGH grouped the chemotherapy regimen. HYM performed data analysis. YJB and WHK collected clinicopathological information from patients, constructed tissue array blocks, and conducted IHC and ISH.

\section{Ethics approval and consent to participate}

The present study was approved by the Institutional Review Board/Independent Ethics Committee of Seoul National University Hospital (H-1706-105-860). The requirement for written consent was waived.

\section{Patient consent for publication}

Not applicable.

\section{Competing interests}

The authors declare that they have no competing interests.

\section{References}

1. Bray F, Ferlay J, Soerjomataram I, Siegel RL, Torre LA and Jemal A: Global cancer statistics 2018: GLOBOCAN estimates of incidence and mortality worldwide for 36 cancers in 185 countries. CA Cancer J Clin 68: 394-424, 2018.

2. Chen W, Wang J, Li X, Li J, Zhou L, Qiu T, Zhang M and Liu P: Prognostic significance of BRCA1 expression in gastric cancer. Med Oncol 30: 423, 2013.

3. Brose MS, Rebbeck TR, Calzone KA, Stopfer JE, Nathanson KL and Weber BL: Cancer risk estimates for BRCA1 mutation carriers identified in a risk evaluation program. J Natl Cancer Inst 94: 1365-1372, 2002.

4. Wang L, Wei J, Qian X, Yin H, Zhao Y, Yu L, Wang T and Liu B: ERCC1 and BRCA1 mRNA expression levels in metastatic malignant effusions is associated with chemosensitivity to cisplatin and/or docetaxel. BMC Cancer 8: 97, 2008.

5. Semba S, Yokozaki H, Yasui W and Tahara E: Frequent microsatellite instability and loss of heterozygosity in the region including BRCA1 (17q21) in young patients with gastric cancer. Int J Oncol 12: 1245-1251, 1998.

6. Shim HJ, Yun JY, Hwang JE, Bae WK, Cho SH, Lee JH Kim HN, Shin MH, Kweon SS, Lee JH, et al: BRCA1 and XRCC1 polymorphisms associated with survival in advanced gastric cancer treated with taxane and cisplatin. Cancer Sci 101: $1247-1254,2010$

7. Goffin JR, Chappuis PO, Bégin LR, Wong N, Brunet JS, Hamel N, Paradis AJ, Boyd $\mathbf{J}$ and Foulkes WD: Impact of germline BRCA1 mutations and overexpression of p53 on prognosis and response to treatment following breast carcinoma: 10-year follow up data. Cancer 97: 527-536, 2003.

8. Kennedy RD, Quinn JE, Mullan PB, Johnston PG and Harkin DP: The role of BRCA1 in the cellular response to chemotherapy. J Natl Cancer Inst 96: 1659-1668, 2004.

9. Reguart N, Cardona AF, Carrasco E, Gomez P, Taron M and Rosell R: BRCA1: A new genomic marker for non-small-cell lung cancer. Clin Lung Cancer 9: 331-339, 2008.

10. Aly A and Ganesan S: BRCA1, PARP, and 53BP1: Conditional synthetic lethality and synthetic viability. J Mol Cell Biol 3: 66-74, 2011.

11. Foulkes WD: BRCA1 and BRCA2: Chemosensitivity, treatment outcomes and prognosis. Fam Cancer 5: 135-142, 2006.

12. Bolton KL, Chenevix-Trench G, Goh C, Sadetzki S, Ramus SJ, Karlan BY, Lambrechts D, Despierre E, Barrowdale D, McGuffog L, et al: Association between BRCA1 and BRCA2 mutations and survival in women with invasive epithelial ovarian cancer. JAMA 307: 382-390, 2012.

13. Kim JW, Cho HJ, Kim M, Lee KH, Kim MA, Han SW, Oh DY, Lee HJ, Im SA, Kim TY, et al: Differing effects of adjuvant chemotherapy according to BRCA1 nuclear expression in gastric cancer. Cancer Chemother Pharmacol 71: 1435-1443, 2013.

14. Wei J, Costa C, Ding Y, Zou Z, Yu L, Sanchez JJ, Qian X, Chen H, Gimenez-Capitan A, Meng F, et al: mRNA expression of BRCA1, PIAS1, and PIAS4 and survival after second-line docetaxel in advanced gastric cancer. J Natl Cancer Inst 103: 1552-1556, 2011.

15. Zhang ZZ, Liu YJ, Yin XL, Zhan P, Gu Y and Ni XZ: Loss of BRCA1 expression leads to worse survival in patients with gastric carcinoma. World J Gastroenterol 19: 1968-1974, 2013. 
16. Edge SB, Byrd DR, Compton CC, Fritz AG, Greene FL and Trotti A: AJCC cancer staging manual, 7th edition. France: Springer, 2010

17. Livak KJ and Schmittgen TD: Analysis of relative gene expression data using real-time quantitative PCR and the 2(-Delta Delta C(T)) method. Methods 25: 402-408, 2001

18. Tsibulak I, Wieser V, Degasper C, Shivalingaiah G, Wenzel S, Sprung S, Lax SF, Marth C, Fiegl H and Zeimet AG: BRCA1 and BRCA2 mRNA-expression prove to be of clinical impact in ovarian cancer. Br J Cancer 119: 683-692, 2018.

19. Al-Mulla F, Abdulrahman M, Varadharaj G, Akhter N and Anim JT: BRCA1 gene expression in breast cancer: A correlative study between real-time RT-PCR and immunohistochemistry. J Histochem Cytochem 53: 621-629, 2005.

20. Garg K, Levine DA, Olvera N, Dao F, Bisogna M, Secord AA, Berchuck A, Cerami E, Schultz N and Soslow RA: BRCA1 immunohistochemistry in a molecularly characterized cohort of ovarian high-grade serous carcinomas. Am J Surg Pathol 37: $138-146,2013$

21. Peshkin BN, Alabek ML and Isaacs C: BRCA1/2 mutations and triple negative breast cancers. Breast Dis 32: 25-33, 2010.

22. Galizia E, Giorgetti G, Piccinini G, Santinelli A, Loretelli C, Bianchi F, Gagliardini D, Carbonari G, Pisa E, Belvederesi L, et al: BRCA1 expression in triple negative sporadic breast cancers. Anal Quant Cytol Histol 32: 24-29, 2010.

23. Wang GH, Zhao CM, Huang Y, Wang W, Zhang S and Wang X: BRCA1 and BRCA2 expression patterns and prognostic significance in digestive system cancers. Hum Pathol 71: 135-144, 2018.
24. Meijer TG, Verkaik NS, Sieuwerts AM, van Riet J, Naipal KAT, van Deurzen CHM, den Bakker MA, Sleddens HFBM, Dubbink HJ, den Toom TD, et al: Functional ex vivo assay reveals homologous recombination deficiency in breast cancer beyond BRCA gene defects. Clin Cancer Res 24: 6277-6287, 2018.

25. Clark-Knowles KV, O'Brien AM and Weberpals JI: BRCA1 as a therapeutic target in sporadic epithelial ovarian cancer. J Oncol 2010: 891059, 2010

26. Pothuri B: BRCA1- and BRCA2-related mutations: Therapeutic implications in ovarian cancer. Ann Oncol 24 (Suppl 8): viii22-viii27, 2013.

27. Yang Q, Sakurai T, Mori I, Yoshimura G, Nakamura M, Nakamura Y, Suzuma T, Tamaki T, Umemura T and Kakudo K: Prognostic significance of BRCA1 expression in Japanese sporadic breast carcinomas. Cancer 92: 54-60, 2001.

28. Zhang Y, Wu H, Yang F, Ning J, Li M, Zhao C, Zhong S, $\mathrm{Gu} \mathrm{K}$ and Wang H: Prognostic value of the expression of DNA repair-related biomarkers mediated by alcohol in gastric cancer patients. Am J Pathol 188: 367-377, 2018.

29. Yoshida K and Miki Y: Role of BRCA1 and BRCA2 as regulators of DNA repair, transcription, and cell cycle in response to DNA damage. Cancer Sci 95: 866-871, 2004.

30. Takata M, Tachiiri S, Fujimori A, Thompson LH, Miki Y, Hiraoka M, Takeda S and Yamazoe M: Conserved domains in the chicken homologue of BRCA2. Oncogene 21: 1130-1134, 2002. International (CC BY-NC-ND 4.0) License. 